Case Report

\title{
Dramatic response of CTNNB1 and VEGFR-2 mutant temporal bone squamous cell carcinoma to bevacizumab in combination with pemetrexed
}

\author{
Lai Wei ${ }^{1, *}$, Lizhi Wang ${ }^{1, *}$, Ziye Liu ${ }^{1}$, Meiyi Wang ${ }^{1}$, Weili Lu' ${ }^{1}$, Dewei Zhao ${ }^{2}$, Bin Yang ${ }^{2}$, \\ Xuejun Kong ${ }^{3}$, Yan Ding ${ }^{2,3,4}$ and Zhiqiang Wang ${ }^{1}$ \\ ${ }^{1}$ Department of Otolaryngology, Affiliated Zhongshan Hospital of Dalian University, Dalian, China \\ ${ }^{2}$ The Institute for Translational Medicine, Affiliated Zhongshan Hospital of Dalian University, Dalian, China \\ 3 Department of Pediatrics, Children's Hospital of Boston, Harvard Medical School, Boston, MA, USA \\ ${ }^{4}$ Program for Autism Research, Massachusetts General Hospital, Harvard Medical School, Boston, MA, USA \\ * These authors have contributed equally to this work \\ Correspondence to: Zhiqiang Wang, email:wzqwlzwl@sohu.com \\ Yan Ding, email: yan.ding@childrens.harvard.edu \\ Keywords: temporal bone squamous cell carcinoma, recurrent tumor, targeted therapy \\ Received: November 23, $2016 \quad$ Accepted: July 18, $2017 \quad$ Published: July 28, 2017
}

Copyright: Wei et al. This is an open-access article distributed under the terms of the Creative Commons Attribution License 3.0 (CC BY 3.0), which permits unrestricted use, distribution, and reproduction in any medium, provided the original author and source are credited.

\section{ABSTRACT}

High recurrence rates and poor survival rates for late stage/advanced temporal bone squamous cell carcinoma with the standard treatments continues to be a significant challenge to otolaryngologists. Targeted therapy for temporal bone squamous cell carcinoma after relapse has not been reported. Here we present a 58-year-old man who was diagnosed with recurrent temporal bone squamous cell carcinoma and treated with a regimen developed using whole exome sequencing. Somatic mutations in genes encoding catenin beta 1 and vascular endothelial growth factor receptor 2 were identified in the patient's tumor sample compared to the normal tissue. The patient was then treated with Bevacizumab in combination with pemetrexed. After two weeks of treatment, tumor volume was reduced by $\mathbf{9 5 \%}$ measured by MRI, and the Visual Analogue Scale headache scores went down from $10 / 10$ to $2 / 10$. Our results reveal novel gene mutations of temporal bone squamous cell carcinoma and demonstrate, for the first time, an effective targeted therapy for temporal bone squamous cell carcinoma. The successful treatment regimen of bevacizumab and pemetrexed may provide a new treatment option for treating recurrent temporal bone squamous cell carcinoma that fails to respond to conventional tumor resection, radiotherapy, and/or chemotherapy.

\section{INTRODUCTION}

Temporal bone squamous cell carcinoma (TBSCC) is uncommon, accounting for fewer than $0.2 \%$ of all tumors of the head and neck with an incidence of 1 to 6 per one million [1]. Only 200 new cases of temporal bone cancer may be diagnosed each year across the United States. This number includes cancers arising from skin of the pinna that spread to the temporal bone; primary tumors of the external auditory canal (EAC), middle ear, mastoid, or petrous apex; and metastatic lesions to the temporal bone. TBSCC accounts for $80 \%$ of all temporal bone tumors [2]. Formulating an optimal evaluation and treatment protocol for TBSCC continues to be a significant challenge to otolaryngologists due to its rare incidence and the complexity of the anatomy in the region [3]. Surgical resection combined with postoperative radiation therapy has been described as the standard of care for primary site TBSCC [4]. However, recurrence rate of a late stage aggressive subtype TBSCC is significantly high [5]. Recently, targeted therapy has been successfully used in a number of different cancers such as cervical cancer, 
non-small cell lung cancer, olfactory neuroblastoma, and head \& neck cancers [6-11]. However, to the best of our knowledge, targeted therapy for TBSCC based on individual genomic profile has never been reported.

In the present case, a patient diagnosed with TBSCC had received resection of left temporal bone followed by radiotherapy and chemotherapy. He presented with a recurrence of TBSCC three months after surgical treatment. Identification of genomic variations in the tumor tissue made via whole exome sequencing (WES) led to the development of a treatment regimen of bevacizumab combining with pemetrexed. We report herein the first exceptional therapeutic response to bevacizumab targeted therapy in combination with pemetrexed chemotherapy in a multiply recurrent TBSCC with genetically confirmed vascular endothelial growth factor receptor 2 (VEGFR-2) and catenin beta 1 (CTNNB1) mutation.

\section{CASE REPORT}

A 58-year-old male was diagnosed with left maxillary sinus squamous cell carcinoma and underwent a surgical operation in September 2013. He complained of nasal obstruction, facial swelling and intermittent epistaxis three months later. Dark red neoplasm located in the patient left nasal cavity was observed. CT scan showed the invasion of multiple structures including anterior left frontal sinus, ethmoid sinus, maxillary sinus, nasal septum, pterygopalatine fossa and hard palate. The patient received tumor radical excision and confirmed to be squamous cell carcinoma by pathological examination.

After the surgery, the patient received radiotherapy of $70 \mathrm{~Gy}$ in fractions of $2 \mathrm{~Gy}$. However, one year and five months after post-operative treatment, the patient presented with complaints of intermittent epistaxis and decreased vision of the left eye for two months. Examination showed that he had loss of vision and a tumor was located on the top wall of the left residual hard palate. The patient agreed to receive extended resection of the left maxilla. After that, he received radiotherapy of 50 Gy in fractions of $1.3 \mathrm{~Gy}$ and 5 courses of chemotherapy, including paclitaxel (IV, once every three weeks, 240mg at a time) $\left(135 \mathrm{mg} / \mathrm{m}^{2}\right)$ and nedaplatin (IV, once every three weeks, $140 \mathrm{mg}$ at a time $)\left(80 \mathrm{mg} / \mathrm{m}^{2}\right)$. The complication of leukopenia was observed and it was recovered after using recombinant human granulocyte colony-stimulating factor. Oral ulcer healed, but hair losing because of early chemotherapy. Nausea and vomiting were disappeared after injection of antipathectic.

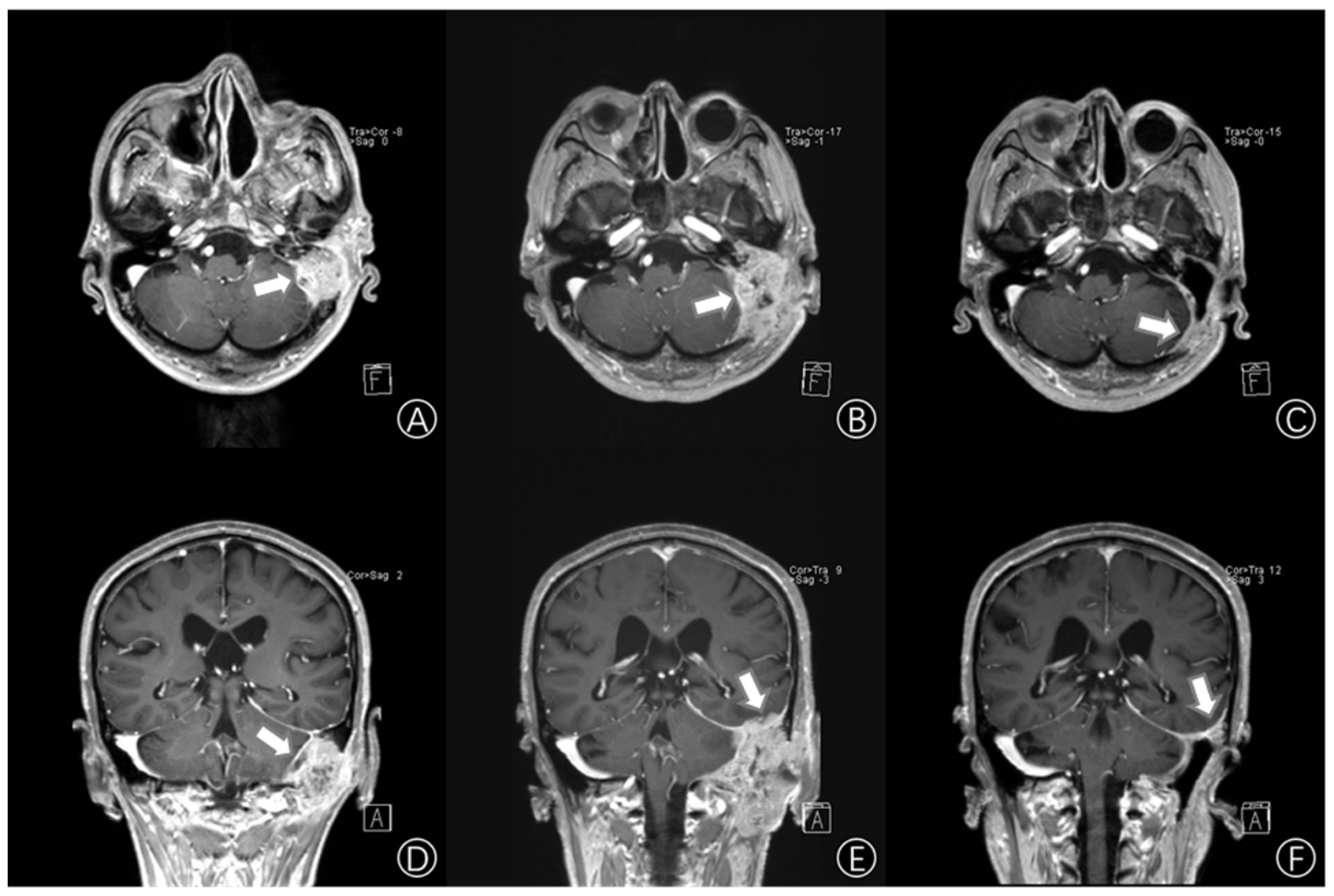

Figure 1: Imaging examination. A. and D. Enhancement cerebral MRI showing the left temporal lobe space-occupying lesion (white arrows) (size: $27.5 \mathrm{~mm} \times 38.0 \mathrm{~mm} \times 40.5 \mathrm{~mm}$ ). B. and E. Three months after the first middle ear surgery, enhancement cerebral MRI shows the tumor (white arrows) is recurrent (size: $40.5 \mathrm{~mm} \times 52.3 \mathrm{~mm} \times 63.6 \mathrm{~mm}$ ). C. and F. Two weeks after bevacizumab and pemetrexed treatment, an enhancement cerebral MRI shows that the right temporal lobe space occupying lesion has reduced by $95 \%$ (size: $13.2 \mathrm{~mm} \mathrm{x}$ 20.5mm x 27.0mm). (A, B, C. Horizontal enhancement imaging. D, E, F. Coronal enhancement imaging). 
Table 1: The Significantly Mutated Genes from the Tumor Tissue of the Patient Detected by Whole Exome Sequencing

\begin{tabular}{|l|l|l|l|l|l|}
\hline Gene name & Exon & Amino Acid Change & Nucleotide Change & Genotype & Mutation type \\
\hline CTNNB1 & 3 th & p.Met12Leu & c.34A $>$ C & heterozygosis & Missense mutation \\
\hline VEGFR-2 & 11 th & p.Gln472His & c.1416A $>$ T & homozygosis & Missense mutation \\
\hline
\end{tabular}

Three years after the first surgery, the patient came back and complained of hearing loss and otorrhea for 10 months. White neoplasm located at the patient left EAC was observed. CT scans showed the invasion of multiple structures including EAC, tympanic cavity, mastoid and occipital. MRI distinctly showed that there was a lesion occupying the left temporal bone nearby the left middle fossa of skull (size: $27.5 \mathrm{~mm} \times 38.0 \mathrm{~mm}$ $\mathrm{x} 40.5 \mathrm{~mm}, \mathrm{~T} 4)$ (Figure 1A, 1D). The tumor tissues were confirmed as TBSCC by using H\&E staining and Immunohistochemistry (Figure 2).

Then, after radical resection of the tumor for 3 months, it recurred and invaded the left cerebella hemisphere and temporal lobe (size: $40.5 \mathrm{~mm}$ x $52.3 \mathrm{~mm} \times$ $63.6 \mathrm{~mm}, \mathrm{~T} 4)$ by MRI scan (Figure 1B, 1E). That made removing the recurrent tumor more difficult and risky.

Given the failure of tumor resection, we decided to explore the options for targeted therapy for this patient.
WES was employed to detect the target gene mutations from patient tumor/normal tissue pairs on the Illumina NextSeq500 sequencing platform and using a TruSeq Rapid Capture Exome Kit for library construction. The WES data was then analyzed using OncoDecoder ${ }^{\mathrm{TM}}$ (Genomic Future, Inc.). The significantly mutated cancer-related genes that were identified in the tumor tissue compared to the adjacent normal control included CTNNB1 and VEGFR-2 (Table 1).

Specifically, we found a missense mutation p.Met12Leu in exon3 of CTNNB1 and missense mutation p.Gln472His(exon11) in VEGFR-2. To detect the gene expression level of VEGFR-2 and its ligand vascular endothelial growth factor(VEGF), quantitative real time PCR on mRNA extracted from both tumor and normal tissues was carried out, and results showed significant overexpression of both VEGF and VEGFR-2 genes in the tumor tissue compared to the normal tissue (Figure 3).

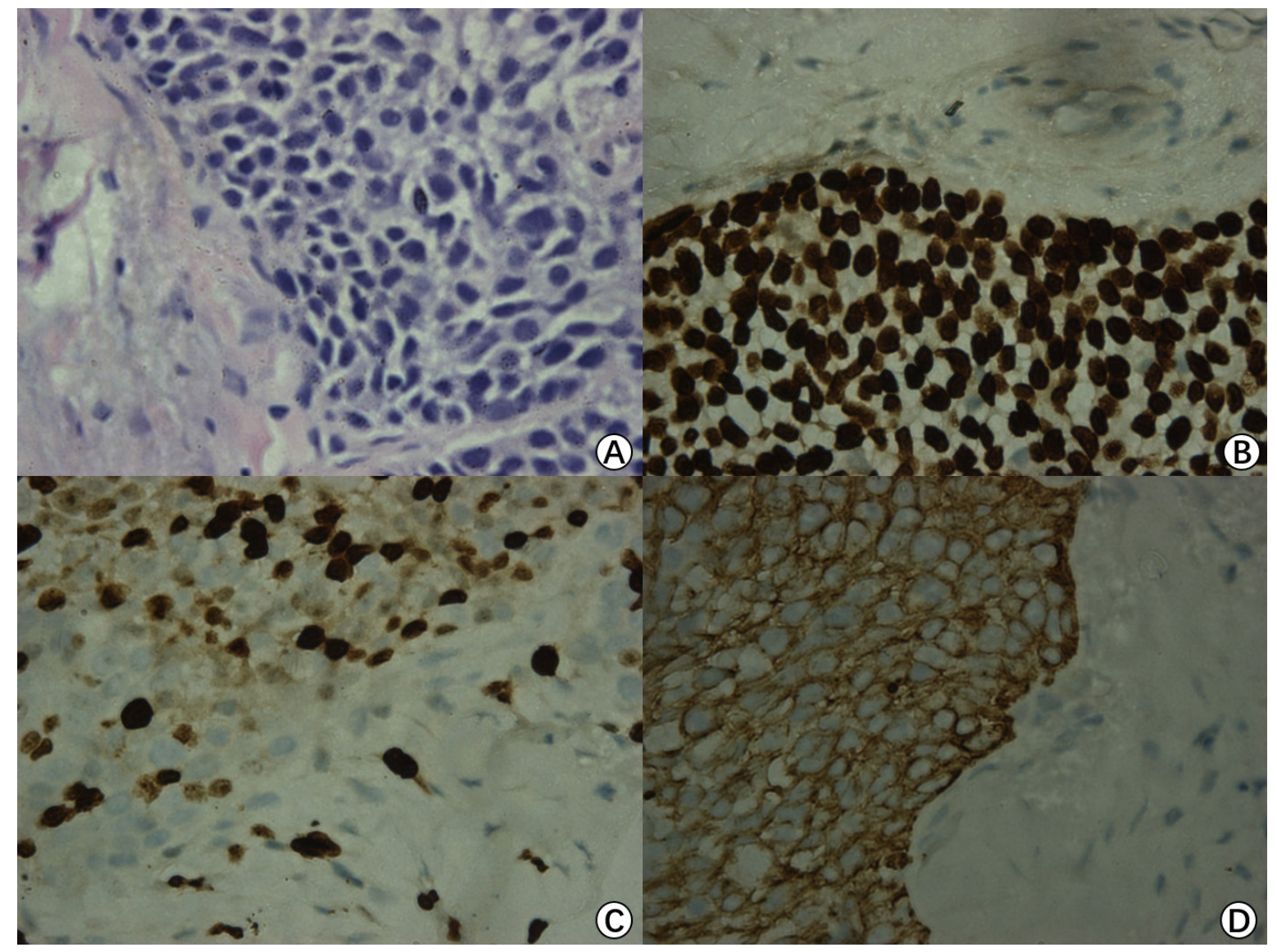

Figure 2: Pathological examination. H\&E staining $(\times 400)$ showed some pink cytoplasm with distinct cell borders and intercellular bridges characteristic A.. Immunohistochemical results show thatP63 B., Ki67 C. and CK516 D. are positive (x400). 
Based on these findings, we carefully screened currently available targeted drugs that may potentially be used for treatment of head and neck cancers. We determined a treatment regimen of bevacizumab and pemetrexed. The ethics committee of the Affiliated Zhongshan Hospital of Dalian University approved the study (The Institutional Review Board approval number is 2016102). The patient received concurrent Bevacizumab (IV, one time per two weeks, 300mg each time for 2 weeks) treatment and pemetrexed (IV, one time a day, $890 \mathrm{mg}$ per 21 days for 4 weeks) treatment. The patient was found in slightly red erythra with itch, and these symptoms resolved spontaneously within 48 hours. Fifteen days later, MRI revealed that the lesion occupying the left temporal lobe space had significantly shrunk by $95 \%$ to a small hypointense area (size $13.2 \mathrm{~mm} \times 20.5 \mathrm{~mm}$ x 27.0mm, T4) (Figure 1C, 1F). Visual Analogue Scale scores of headaches were from $10 / 10$ to $2 / 10$. On targeted therapy Day 35, the patient received extended resection of the temporal bone and reconstruction. Two months later, the patient remains free of new symptoms. Long-term follow-up care has been established for the patient.

\section{DISCUSSION}

A combination of open surgery, radiotherapy and/ or chemotherapy has been considered as standard care for TBSCC. However, it was deemed to have a negative outcome and prognosis. Dean NR et al. reported that the rate of recurrence in TBSCC is close to $34 \%$ [12].

Takenaka, Y et al. reported that Paclitaxol, Cisplatin and 5-fluorouracil are some of the common chemotherapeutic medicine used in squamous cell carcinoma of the EAC [4].Unfortunately, the traditional treatment regimens often fail for recurrent and metastatic TBSCC cases, especially with stage T3-4 [13]. Moreover, some patients may refuse to take the standard protocols due to their intolerance of radiation and/or chemotherapy. Most recently, genome-based precision medicine has drawn a great deal of attention from oncologists. Several studies have shown that the use of targeted medicines can result in either a complete response or a significant improvement in the life quality of patients with a variety of cancers [16]. It is widely accepted that one of the biggest advantages of targeted drugs is their high specificity and low toxicity. To the best of our knowledge, personalized treatment of TBSCC with targeted drugs based on the patient's genomic variations has not been reported.

To explore the opportunity and potential benefit of targeted therapy for advanced and recurrent TBSCC, we sequenced the whole exomes of the tumor/normal tissues of the patient in our genetic test lab and identified several novel significantly mutated cancer genes that may be related to TBSCC including CTNNB1 and VEGFR-2 (Table 1). A few of gene mutation and/expression dysregulation including p16, TP53 mutation, epidermal growth factor receptor (EGFR), pSTAT3, and relaxin-2 have been reported that may be related to the development or progression of different categories of TBSCC [14]. For example, studies have shown overexpression of p53 and EGFR may be valuable biomarkers for identifying TBSCC with high risk of lymph node metastasis [15]. However, in our case, we found novel mutations in CTNNB1 and VEGFR-2 genes that may be related to TBSCC and overexpression of VEGF and VEGFR-2 genes were

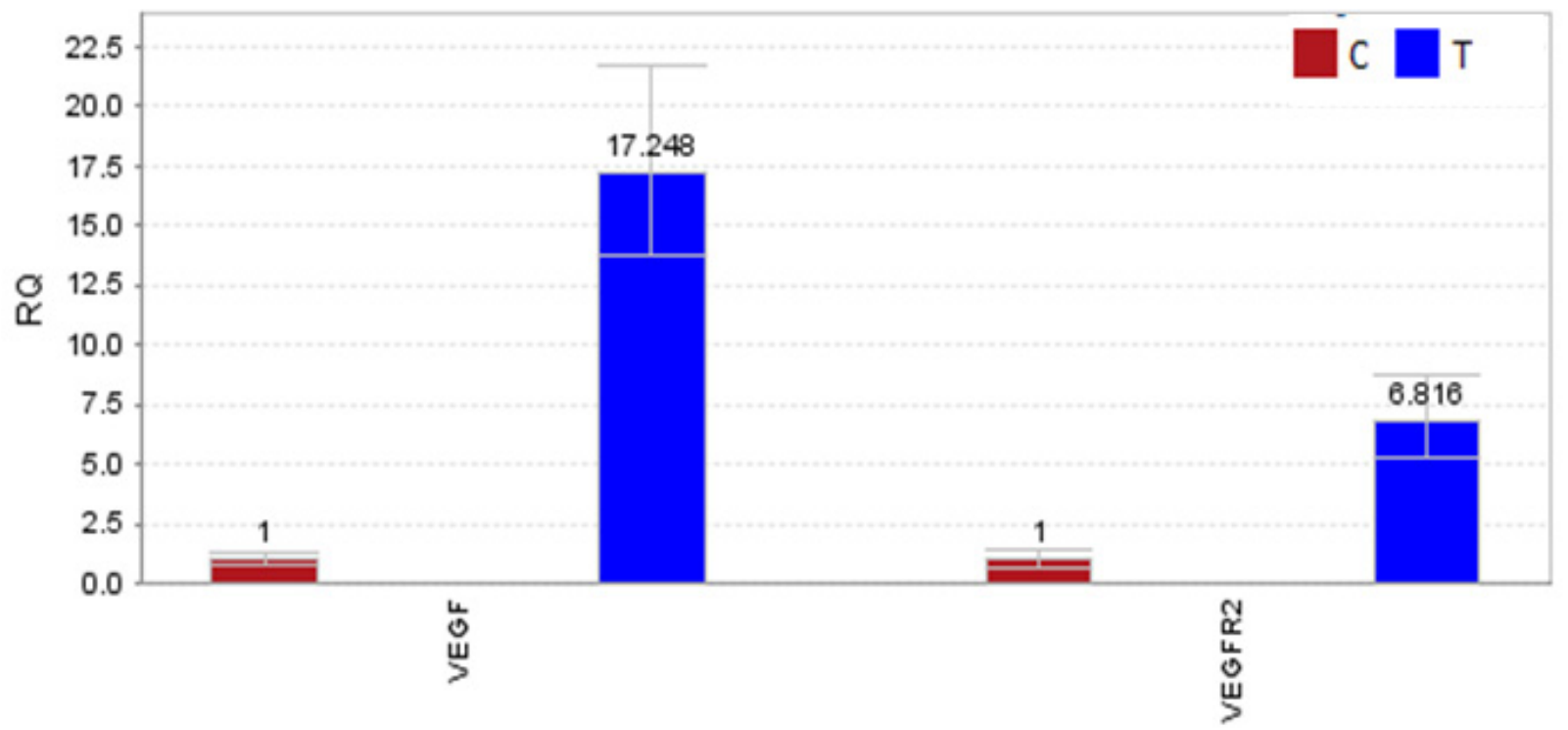

Target

Figure 3: The result of quantitative polymerase chain reaction (qPCR). Overexpression of VEGF and VEGFR-2 in TBSCC tissue compared to normal tissue by qPCR. Expression of VEGF is about 17-fold and VEGFR-2 is about 7-fold higher than normal tissues. 
observed in the tumor tissue. VEGFR-2, also known as vascular endothelial growth factor receptor 2, functions as the main mediator of VEGF-induced angiogenesis in a variety of cancers. Bevacizumab, an anti-vascular endothelial growth factor monoclonal antibody, was the first anti-angiogenesis agent to receive US Food and Drug Administration approval in oncology [16]. Numerous studies of the bevacizumab showed promise in patients with squamous cell carcinoma of the head and neck (SCCHN) $[17,18]$. Nylfot, MJ et al. reported that bevacizumab could reduce tumor proliferative capacity of SCCHN, and combined bevacizumab with cisplatinbased chemo-radiation therapy for SCCHN revealed safe and significant responses [19]. Pemetrexed disodium is a structurally modified folate analogue [20]. It has proven effective in non-small-cell lung cancer and malignant pleural mesothelioma that suppresses both DNA synthesis and folate metabolism [21-23]. In 2011, a result of 40 patients research showed that adding bevacizumab to pemetrexed resulted in promising efficacy outcomes in SCCHN [24]. In our case, genetic tests have shown mutation in VEGFR-2 (Table 1) as well as significant overexpression of VEGFR-2 and VEGF in the patient TBSCC tumor tissue (Figure 3). Since bevacizumab may indirectly blocks VEFGR-2 signaling through inhibiting VEGF, it is perhaps not surprising to see that the patient showed a striking response to a bevacizumab plus pemetrexed treatment regimen (Figure 1). The clinical outcomes from the TBSCC case indicate that VEGFR-2 might be potential target for TBSCC therapy.

TBSCC is an aggressive malignancy with a poor prognosis in advanced cases. The 5-year survival rate for patient with T3-4 was $14.0 \%-55.6 \%[13,25]$. T4 classification, extensive bone involvement and dura involvement was poor predictor of survival rate, separately [26]. Two months after receiving extended resection of the temporal bone and reconstruction, the patient remains free of new symptoms. Shrinking tumors before definitive treatment with surgery/radiation could improve the safety and efficacy of initial treatment, potentially reducing the disabling morbidities that often follow current treatment. As we prepared the final draft of out manuscript, the patient remains in stable state. We recognized the limitations of our study were based on single and short time follow-up case, therefore, it is not clear at this point of time that the targeted therapy would prolong the overall survival of the rare malignant tumor.

$\mathrm{CT}$ of the temporal bone is the standard for assessing bone erosion, while MRI, provides detailed soft tissue resolution, is superior to $\mathrm{CT}$ in diagnosis of temporal bone malignancy that invades the intracranial space and brain tissue and evaluation of the major blood vessels of the skull base. In this case, the patient only receives MRI scanning after radical resection of the malignancy because of the hazards of CT scanning and the advantages of MRI.

Follow-up care has been established for the patient and an update of clinical outcomes is warranted. As TBSCC is a rare disease, collaborative efforts for a cohort study is desirable to further prove the effectiveness of targeted therapy for TBSCC.

\section{CONCLUSIONS}

Surgery followed by chemo-radiotherapy is the current standard of care for TBSCC and fails frequently with a high risk of recurrence and adverse effects. Genome-based targeted therapy for recurrent and late stage TBSCC is potentially an option in terms of its promising clinical response, and hence deserves further investigation in a prospective clinical trial.

\section{Abbreviations}

TBSCC: Temporal bone squamous cell carcinoma; WES: whole exome sequencing; CTNNB1: catenin beta 1; EAC: external auditory canal; VEGFR-2: vascular endothelial growth factor receptor 2; VEGF: vascular endothelial growth factor; EGFR: epidermal growth factor receptor; SCCHN: squamous cell carcinoma of the head and neck.

\section{ACKNOWLEDGMENTS AND FUNDING}

We thank members of the Ding laboratory for advice and support. This work is supported by an Innovative Grant from Chinese Central Government of Human Resources (YD), a Merit Grant for Extraordinary Oversea Talent from Dalian Government (YD), and a Start-up Research Fund from the Affiliated Zhongshan Hospital of Dalian University (YD).

\section{CONFLICTS OF INTEREST}

The authors declare no conflicts of interest.

\section{REFERENCES}

1. Xie B, Zhang T, Dai C. Survival outcomes of patients with temporal bone squamous cell carcinoma with different invasion patterns. Head Neck. 2015; 37: 188-196.

2. Zhang T, Dai C, Wang Z. The misdiagnosis of external auditory canal carcinoma. Eur Arch Otorhinolaryngol. 2013; 270: 1607-1613.

3. Ogawa K, Nakamura K, Hatano K, Uno T, Fuwa N, Itami J, Kojya S, Nakashima T, Shinhama A, Nakagawa T, Toita T, Sakai M, Kodaira T, et al. Treatment and prognosis of squamous cell carcinoma of the external auditory canal and middle ear: a multi-institutional retrospective review of 87 patients. Int J Radiat Oncol Biol Phys. 2007; 68: 13261334. 
4. Takenaka Y, Cho H, Nakahara S, Yamamoto Y, Yasui $\mathrm{T}$, Inohara H. Chemoradiation therapy for squamous cell carcinoma of the external auditory canal: A meta-analysis. Head Neck. 2015; 37: 1073-1080.

5. Zanoletti E, Marioni G, Franchella S, Lovato A, Giacomelli L, Martini A, Mazzoni A. Recurrent squamous cell carcinoma of the temporal bone: critical analysis of cases with a poor prognosis. Am J Otolaryngol. 2015; 36: 352355 .

6. Wang L, Ding Y, Wei L, Zhao D, Wang R, Zhang Y, Gu $X$, Wang Z. Recurrent Olfactory Neuroblastoma Treated With Cetuximab and Sunitinib: A Case Report. Medicine (Baltimore). 2016; 95: e3536.

7. Bizzarri N, Ghirardi V, Alessandri F, Venturini PL, Valenzano Menada M, Rundle S, Maggiore LR, Ferrero S. Bevacizumab for the treatment of cervical cancer. Expert Opin Biol Ther. 2016; 16: 407-419.

8. Schefter T, Winter K, Kwon JS, Stuhr K, Balaraj K, Yaremko BP, Small W Jr, Sause W, Gaffney D. RTOG 0417: efficacy of bevacizumab in combination with definitive radiation therapy and cisplatin chemotherapy in untreated patients with locally advanced cervical carcinoma. Int J Radiat Oncol Biol Phys. 2014; 88: 101-105.

9. Bonomi PD, Mace J, Mandanas RA, Min M, Olsen M, Youssoufian H, Katz TL, Sheth G, Lee HJ. Randomized phase II study of cetuximab and bevacizumab in combination with two regimens of paclitaxel and carboplatin in chemonaive patients with stage IIIB/IV nonsmall-cell lung cancer. J Thorac Oncol. 2013; 8: 338-345.

10. Schefter TE, Winter K, Kwon JS, Stuhr K, Balaraj K, Yaremko BP, Small W Jr, Gaffney DK. A phase II study of bevacizumab in combination with definitive radiotherapy and cisplatin chemotherapy in untreated patients with locally advanced cervical carcinoma: preliminary results of RTOG 0417. Int J Radiat Oncol Biol Phys. 2012; 83: 11791184.

11. Sattler M, Abidoye O, Salgia R. EGFR-targeted therapeutics: focus on SCCHN and NSCLC. ScientificWorldJournal. 2008; 8: 909-919.

12. Dean NR, White HN, Carter DS, Desmond RA, Carroll WR, McGrew BM, Rosenthal EL. Outcomes following temporal bone resection. Laryngoscope. 2010; 120: 15161522.

13. Morita S, Homma A, Nakamaru Y, Sakashita T, Hatakeyama H, Kano S, Fukuda A, Fukuda S. The Outcomes of Surgery and Chemoradiotherapy for Temporal Bone Cancer. Otol Neurotol. 2016; 37: 1174-1182.

14. Marioni G, Martini A, Favaretto N, Franchella S, Cappellesso R, Marino F, Blandamura S, Mazzoni A, Zanoletti E. Temporal bone carcinoma: a first glance beyond the conventional clinical and pathological prognostic factors. Eur Arch Otorhinolaryngol. 2015; 273:2903-10.

15. Morita S, Nakamaru Y, Homma A, Yasukawa S, Hatakeyama H, Sakashita T, Kano S, Fukuda A, Fukuda
S. Expression of p53, p16, cyclin D1, epidermal growth factor receptor and Notch1 in patients with temporal bone squamous cell carcinoma. International Journal of Clinical Oncology. 2016: 1-9.

16. Fury MG, Lee NY, Sherman E, Lisa D, Kelly K, Lipson B, Carlson D, Stambuk H, Haque S, Shen R, Kraus D, Shah J, Pfister DG. A phase 2 study of bevacizumab with cisplatin plus intensity-modulated radiation therapy for stage III/IVB head and neck squamous cell cancer. Cancer. 2012; 118: 5008-5014.

17. Argiris A, Kotsakis AP, Hoang T, Worden FP, Savvides P, Gibson MK, Gyanchandani R, Blumenschein GR Jr, Chen HX, Grandis JR, Harari PM, Kies MS, Kim S. Cetuximab and bevacizumab: preclinical data and phase II trial in recurrent or metastatic squamous cell carcinoma of the head and neck. Ann Oncol. 2013; 24: 220-225.

18. Yamatodani T, Holmqvist B, Kjellen E, Johnsson A, Mineta H, Wennerberg J. Using intravital microscopy to observe bevacizumab-mediated anti-angiogenesis in human head and neck squamous cell carcinoma xenografts. Acta Otolaryngol. 2012; 132: 1324-1333.

19. Nyflot MJ, Kruser TJ, Traynor AM, Khuntia D, Yang DT, Hartig GK, McCulloch TM, Wiederholt PA, Gentry LR, Hoang T, Jeraj R, Harari PM. Phase 1 trial of bevacizumab with concurrent chemoradiation therapy for squamous cell carcinoma of the head and neck with exploratory functional imaging of tumor hypoxia, proliferation, and perfusion. Int J Radiat Oncol Biol Phys. 2015; 91: 942-951.

20. Pivot X, Raymond E, Laguerre B, Degardin M, Cals L, Armand JP, Lefebvre JL, Gedouin D, Ripoche V, Kayitalire L, Niyikiza C, Johnson R, Latz J, et al. Pemetrexed disodium in recurrent locally advanced or metastatic squamous cell carcinoma of the head and neck. Br J Cancer. 2001; 85: 649-655.

21. Scagliotti GV, Parikh P, von Pawel J, Biesma B, Vansteenkiste J, Manegold C, Serwatowski P, Gatzemeier U, Digumarti R, Zukin M, Lee JS, Mellemgaard A, Park K, et al. Phase III study comparing cisplatin plus gemcitabine with cisplatin plus pemetrexed in chemotherapy-naive patients with advanced-stage non-small-cell lung cancer. J Clin Oncol. 2008; 26: 3543-3551.

22. Hanna N, Shepherd FA, Fossella FV, Pereira JR, De Marinis F, von Pawel J, Gatzemeier U, Tsao TC, Pless M, Muller T, Lim HL, Desch C, Szondy K, et al. Randomized phase III trial of pemetrexed versus docetaxel in patients with non-small-cell lung cancer previously treated with chemotherapy. J Clin Oncol. 2004; 22: 1589-1597.

23. Vogelzang NJ, Rusthoven JJ, Symanowski J, Denham C, Kaukel E, Ruffie P, Gatzemeier U, Boyer M, Emri S, Manegold C, Niyikiza C, Paoletti P. Phase III study of pemetrexed in combination with cisplatin versus cisplatin alone in patients with malignant pleural mesothelioma. J Clin Oncol. 2003; 21: 2636-2644.

24. Argiris A, Karamouzis MV, Gooding WE, Branstetter BF, 
Zhong S, Raez LE, Savvides P, Romkes M. Phase II trial of pemetrexed and bevacizumab in patients with recurrent or metastatic head and neck cancer. J Clin Oncol. 2011; 29: 1140-1145.

25. Chi FL, Gu FM, Dai CF, Chen B, Li HW. Survival outcomes in surgical treatment of 72 cases of squamous cell carcinoma of the temporal bone. Otol Neurotol. 2011; 32: 665-669.
26. Mazzoni A, Danesi G, Zanoletti E. Primary squamous cell carcinoma of the external auditory canal: surgical treatment and long-term outcomes. Acta Otorhinolaryngol Ital. 2014; 34: 129-137. 